\title{
Determinación de campos areales de precipitación y evapotranspiración en la margen izquierda de la cuenca del Paraná en territorio de Argentina. I: polígonos de thiessen y kriging
}

\author{
Assessment of domain areas for precipitation and \\ evapotranspiration on the left bank of the Paraná \\ watershed at Argentine territory. I: Thiessen polygons \\ and kriging
}

HÄMMERLY, R.C. ${ }^{1}$, PARIS, R.C. ${ }^{1}$, PAZ-GONZÁLEZ, A. ${ }^{2}$

${ }^{1}$ Facultad de Ingeniería y Ciencias Hídricas (FICH), Universidad del Litoral (UNL), Ciudad Universitaria - CC 217. Ruta 168 - km 472,4 3000 Santa Fe (Argentina).

${ }^{2}$ Centro de Investigaciones Científicas Avanzadas (CICA) - Facultad de Ciencias, grupo AQUATERRA, Universidade da Coruña. Campus Elviña As Carballeiras s/n 15071 A Coruña (España).

*Contacto: rhammer@fich.unl.edu.ar

https://doi.org/10.17979/cadlaxe.2019.41.0.5818 enviado: $2 / 2 / 2019$ aceptado $7 / 10 / 2019$

\section{Resumen}

La precipitación (P) y la evapotranspiración potencial (ETP) son términos de la ecuación del balance hídrico que se miden en estaciones locales; sin embargo el uso de modelos de estimación de caudal requiere datos de los mismos a escala de cuenca. En este trabajo se presen- 
tan los resultados de la estimación de la precipitación y la evapotranspiración, interpolados mediante polígonos de Thiessen y la técnica geoestadística de kriging en aquellas subcuencas de la margen izquierda del Río Paraná, en territorio argentino que disponen de registros de caudal. Se estudiaron doce cuencas ubicadas en las provincias de Misiones, Corrientes y Entre Ríos, y se usaron las series de datos meteorológicas disponibles dentro de las mismas y en su periferia, en un periodo común de tiempo, entre 1970 y 2010. Previa a la obtención de la precipitación media areal se completan los datos mensuales faltantes. A partir de dicha información se pudo ponderar la aportación espacial de cada sitio de medida. Los datos medidos mensuales de precipitación y ETP presentaron dependencia espacial que fue descrita por semivariogramas de tipo lineal y gaussiano, respectivamente. En comparación con los polígonos de Thiessen, el krigeado o kriging ordinario proporciona estimaciones areales inferiores de precipitación en 11 de las 12 cuencas estudiadas, observándose las mayores diferencias en la provincia de Misiones. Se concluye que no se evidencia una mejor estimación de campos de precipitación y de ETP como resultado del incremento de la complejidad del método usado para la obtención de los mismos

Palabras clave: variabilidad espacial, campos areales, precipitación, evapotranspiración potencial, cuenca del Paraná.

\begin{abstract}
Precipitation (P) and potential evapotranspiration (ETP) are terms of the water balance equation that are at the catchment scale. This work presents results of precipitations and evapotranspiration estimations obtained by interpolations performed both by Thiessen polygons and the kriging geoestatistical technique; the study involves catchments located at the left bank of the Paraná river, within Argentinean territory, having discharge records. Thus, twelve catchments located in the provinces of Misiones, Corrientes and Entre Ríos have been studied. For this purpose the available meteorological data sets recorded during a common period of time, i.e. between 1970 and 2010, within the perimeter and in the external neighborhood of each catchment have been used. Before assessing average precipitation and ETP of the domain areas nonexistent monthly data were approximated form neighbor stations. Then, the spatial contribution of each measurement site to the areal domain has been weighted. Monthly precipitation and ETP showed spatial dependency that was modeled by linear and Gaussian semivariograms, respectively. In comparison with the Thiessen polygon methods ordinary kriging provided lower areal domain estimates of precipitation in 11 out of the 12 basins studied catchments, and the higher differences were observed at those of the Misiones province. It was concluded that increasing the complexity of the method used for estimating areal precipitation or ETP shows no evidence of better estimates of these variables.
\end{abstract}

Key words: spatial variability, domain areas, precipitation, potential evapotranspiration, Paraná catchment. 


\section{INTRODUCCIÓN}

La aplicación de modelos de estimación de caudales de tipo concentrado o agregados a nivel de cuencas, o subcuencas, requiere información de las variables hidrológicas de entrada a los modelos que resuma el comportamiento areal de la misma. Es decir la precipitación que cae sobre la cuenca o la evaporación y evapotranspiración que tiene lugar sobre la misma. Estos datos son registrados en forma puntual en las estaciones, los que luego deben ser tratados para extenderlos al área ocupada por la cuenca de interés. Se pretende entonces conocer en el espacio cuales son los valores que adoptan esas variables de entrada, en un tiempo dado, y en lo posible definir de qué tipo es la relación entre dos sitios de mediciones y cómo puede expresarse en términos funcionales. Esto dependerá de la vinculación o relación entre los sitios y se podrá de este modo determinar cuál es la estructura de variación espacial que presenta.

Los métodos disponibles para la estimación areal de variables climáticas de las que depende el balance hídrico como la precipitación (P) y la evapotranspiración (ET) se basan en premisas muy diferentes. El más simple es la media aritmética de los valores registrados en las estaciones asociadas al área estudiada. En este caso la variación espacial de la precipitación o la evapotranspiración frente a una densidad insuficiente de la red de estaciones que proporcionan datos climatológicos puede llevar a errores considerables, principalmente en zonas montañosas que presentan gran variación espacial de estas variables. Por ello, para evaluar la P y la ET espacialmente sobre una cuenca, subcuenca o cualquier superficie definida a partir de medidas puntuales se suele ponderar la aportación espacial de cada sitio a través de diferentes métodos. El trazado de los polígonos de Thiessen, la regionaliza- ción de estaciones o las técnicas de kriging son métodos que han sido frecuentemente empleados.

La interpolación mediante el método de los polígonos de Thiessen permite calcular medias areales en base a la ubicación relativa de las estaciones; por tanto este es un método geométrico, que no asume ninguna relación de dependencia entre datos medidos entre estaciones vecinas. La regionalización es una metodología que permite generar información en una ubicación, en base a información conocida en otras áreas que posean un comportamiento semejante; por tanto previo a la interpolación mediante este método se requiere definir de modo preciso regiones homogéneas. El kriging es una técnica de interpolación que se basa en el análisis de la estructura geoestadística de variación de la variable estudiada: es decir, el kriging se apoya en el conocimiento del comportamiento de la variable en el espacio, con la premisa de que puntos próximos tendrán valores más parecidos que puntos más alejados, ya que asume que una muestra de puntos de una población se encuentra correlacionada en el espacio (TUCCI, 2002). Además de las técnicas aquí mencionados, existen diversos métodos determinísticos disponibles para la predicción espacial como son la distancia inversa, interpolación polinomial global, interpolación polinomial local, triangulación lineal, funciones de base radial entre otros.

En este punto es conveniente presentar algunas definiciones. Se entiende por "precipitación areal" aquella que cae sobre toda la superficie de la cuenca y resulta sinónimo de superficie media de la cuenca en sentido espacial. Por "precipitación media areal" se considera como aquella precipitación sobre toda la superficie de la cuenca, pero que esta promediada en el tiempo, por ejemplo una serie de años, o una serie de valores del mismo mes. Conceptos similares son considerados para la evapotranspiración. 
También es importante tener en cuenta los conceptos de estadística, en la que se basa el método clásico de interpolación mediante polígonos de Thiessen, usado desde 1911, y de geostadística, cuyo desarrollo a partir de mediados de la década de 1960 llevó a la interpolación mediante diversos tipos de kriging. Partiendo del supuesto que las variables naturales tienen una componente determinística y una aleatoria, la estadística nos proporciona una descripción del campo de observaciones en un determinado estadio, y la geoestadística complementa esta visión, considerando que este campo de observaciones no es único. Matheron, definió inicialmente a la "Geoestadística" como: "la ciencia del estudio estadístico de los procesos geológico", y en su obra de 1971, como "la aplicación de la teoría de las variables regionalizadas a la estimación de procesos o fenómenos geológicos en el espacio" (MATHERON, 1971). Su objetivo fundamental es el análisis de la estructura de correlación de las variables a partir de su consideración como variable regionalizada. Una variable regionalizada se define como una variable aleatoria distribuida en el espacio y con una estructura espacial de variación. El fenómeno representado por éstas se denomina regionalización, o regionalización geoestadística (DAMILANO y PARIS, 2014).

La geoestadística se ocupa de obtener estimaciones de las variables regionalizadas (VR) a partir del conocimiento de su estructura de variación espacial (BARBOSA LANDÍN, 1997). Para ello es necesario conocer cuál es la función que gobierna su ocurrencia o dicho de otro modo cuál es la función (estadística-matemática) que representa esta variación espacial. En la Estadística clásica o convencional, esta función se estima a partir de una muestra (Estadística Inferencial). Así, con la muestra determinada experimentalmente -que se asume re- presentativa de la población en estudio- se calculan los estadísticos, con ellos los parámetros y con estos últimos la ley de probabilidad que ajusta a la distribución de frecuencias observadas: Normal, LogNormal, Gumbel, etc.

El problema, es que en el caso de las variables naturales que se estudian como variables regionalizadas, se cuenta generalmente, o mejor dicho casi siempre, con un resultado único $\mathrm{Z}(\mathrm{xi})$, que son precisamente los valores que se han medido u observado en el área de estudio. Por ello es necesario realizar la inferencia o estimación de su estructura probabilística bajo ciertas hipótesis. Para ello la geoestadística utiliza una restricción llamada hipótesis intrínseca, que permite el uso de los resultados de una VR por medio del método de los momentos. Según la teoría de probabilidad, una serie de $\mathrm{k}$ variables aleatorias (VA) $z_{1}, z_{2}, z_{3}, \ldots, z_{k}$, (en general no independientes) define un vector de VA $\mathrm{o}$ vector aleatorio $\mathrm{z}$ con $\mathrm{k}$ componentes. Cuando el número de componentes tiende a infinito (esto es el número de VA es infinito) $\mathrm{z}$ se llama función aleatoria (FA). Luego, la interpretación probabilística de una VR como una realización de una función aleatoria FA tiene sentido operativo sólo si es posible inferir su función de distribución o ley de probabilidad o al menos los parámetros que caracterizan esta distribución (CARRERA y SAMPER, 1985). Se infiere que se deberían de conocer los parámetros de cada una de las distribuciones de las variables analizadas en cada uno de los puntos de medición y la Geoestadística ofrece herramientas para ello.

Son numerosos los ejemplos de aplicación de kriging para la obtención de curvas de isovalor: DEMEY et al. (1996); MEJÍA et al. (1999); CORTÉZ et al. (2005); IZQUIERDO et al. (2006); ÍNIGUEZ et al. (2011), entre otros. En el caso particular de las curvas isohietas se pueden mencionar los 
resultados logrados por GUERRA et al. (2006) o PINEDA et al. (2011) y concretamente en Argentina MEZHER et al. (2009) y HÄMMERLY et al. (2012), entre otros.

Cualquier técnica de obtención de campos areales puede ser aplicada indistintamente a cualquier variable hidrológica que se desea representar en forma espacial. Así, para la estimación de la precipitación media de la cuenca, es necesario disponer de datos puntuales medidos en estaciones meteorológicas dentro de la misma y su periferia, en un periodo común de tiempo. En relación a las transferencias de agua a la atmósfera, la medición de la evaporación se da por medio de dispositivos instalados en los abrigos, como los evaporímetros, mientras que la evapotranspiración se puede medir en campo mediante lisímetros y en parcelas experimentales, pero su obtención es laboriosa y costosa. Se recurre entonces a la estimación de la evapotranspiración potencial (ETP) a través de fórmulas empíricas en función de datos meteorológicos. Entre las fórmulas más utilizadas se encuentra la de Thornthwaite, pero se le critica que sólo usa datos de temperatura y que suele sobreestimar. Es por ello que se considera más adecuado, en la medida que se disponga de los datos necesarios, utilizar la fórmula de Penman-Montheith, que tiene en cuanta para su cálculo un término radiativo y otro aerodinámico, requiriendo datos de temperatura, velocidad de vientos, heliofanía y tensión de vapor, posibles de obtener de estaciones sinópticas, junto con otros datos de tablas donde se considera la ubicación y la época del año (CHOW, 1996; TUCCI, 2000; UNESCO, 2006).

El Balance Hídrico Superficial fue una de las actividades previstas dentro del Programa Marco para la Gestión Sostenible de los Recursos Hídricos de la Cuenca del Plata, llevado a cabo por el Comité Intergubernamental Coordinador de los Países de la Cuenca del
Plata en relación con los efectos de variabilidad y cambio climático. Este Balance Hídrico Superficial se realizó como parte del Balance Hídrico Integrado, pensado como instrumento de apoyo para la gestión integrada de los recursos hídricos de la Cuenca, teniendo en cuenta la distribución, la calidad, el uso y demanda del agua. Para llevar a cabo estas modelaciones, previamente se probaron diferentes campos de precipitación y evapotranspiración potencial, con la finalidad de comparar los resultados obtenidos en los balances según las diferentes técnicas. En este trabajo se presentan los resultados de precipitación y ETP media areal, en cuencas que disponen de registros de caudales, obtenidos mediante dos métodos: polígonos de Thiessen y kriging. Por ello, el estudio se limita a la margen izquierda del río Paraná, en un total de 12 cuencas, en las que existen los mencionados registros.

\section{MATERIAL Y MÉTODOS}

Las 12 cuencas de la margen izquierda del río Paraná que disponen de registros de caudales y en las que se determinaron campos areales están ubicadas en las provincias de Misiones, Corrientes y Entre Ríos. Las cuencas consideradas tienen una extensión muy variable y pertenecen a los siguientes arroyos y ríos: arroyo Piray Miní, arroyo Piray Guazú, Arroyo Paranay, río Santa Lucía, río Corriente (Paso Lucena), río Batel, río Corriente (los Laureles), río Barrancas, río Guayquiraró, arroyo Feiciano, arroyo Nogoyá y río Gualeguay (HÄMMERLY, 2017).

Se usaron las series de datos meteorológicas disponibles no solo dentro de las cuencas de la margen izquierda del río Paraná estudiadas, sino también las de la periferia de las mismas en territorio argentino, incluyendo algunas la margen derecha, en un periodo común de tiempo, entre 1970 y 2010; en total se utilizaron datos de 84 esta- 
ciones (HÄMMERLY et al., 2018). Previa a la obtención de la precipitación media areal se completan los datos mensuales faltantes. Esto se realiza en el programa CHAC (Cálculo hidrometeorológico de aportaciones y crecidas) empleado para el cálculo del balance hídrico (CEDEX, 2012) a partir de un modelo de regresión bivariado con estacionarización mensual de las series de datos. La elección de la pareja de estaciones que va a ser utilizada para el completado de cada una de las lagunas de una estación dada se basa en considerar un umbral mínimo del coeficiente de correlación múltiple ponderado según la expresión de la matriz de priorización. Se rellenaron las precipitaciones mensuales en aquellas estaciones con datos faltantes, considerando grupos de estaciones cercanazas para el rellenamiento de las series.

El método adoptado para la estimación de la evapotranspiración potencial fue el de Penman - Monteith Para su determinación se requieren datos mensuales de temperatura media $\left({ }^{\circ} \mathrm{C}\right)$, humedad relativa $(\%)$, heliofanía efectiva (hs) y velocidad de viento ( $\mathrm{km} /$ día). En resumen los pasos seguidos para la determinación de la evapotranspiración potencial areal de las subcuencas fueron los siguientes: 1) el rellenamiento de datos mensuales faltantes para cada variable, 2) la confección del archivo de parámetros para el cálculo de la ETP, y 3) el cálculo de la ETP según Penman-Moteith para cada estación, siendo 38 el total de estaciones disponibles.

Para cada estación pluviométrica estudiada se obtuvo una sola serie de valores mensuales de $\mathrm{P}$ promediados durante todo el período de estudio. En aquellas estaciones en que se pudo estimar la ETP se obtuvo otra serie similar de la misma, Finalmente, a partir de dicha información se pudo ponderar la aportación espacial de cada sitio de medida tanto para la precipitación como para la evapotranspiración potencial me- diante dos métodos, polígonos de Thiessen y kriging, lo que corresponde a un modelo agregado, como se describe a continuación.

\section{Interpolación mediante polígonos de} Thiessen

El método de Polígono de Thiessen se basa en ponderar el valor una variable de interés, por ejemplo P o ETP, en cada estación en función de un área de influencia (Ai), cuya superficie que se calcula según un procedimiento de trazado de polígonos. El procedimiento asume que en el área de influencia, definida por la poligonal, ocurre el mismo valor de lluvia de aquel observado en la estación meteorológica más cercana. La Ecuación 1 indica cómo se obtiene la precipitación media por medio de la ponderación.

$$
P m=\frac{\sum_{1}^{n} A i P i}{A}
$$

Siendo:

Pm: precipitación media de la cuenca

Pi: precipitación de la estación i

Ai: área de influencia de la estación i

A: área total de la cuenca

$\mathrm{n}$ : cantidad de estaciones que influyen en la cuenca

La rasterización del dominio bajo análisis y, la posterior estimación de la P y ETP promedio para cada subcuenca supone que la variabilidad de estos componentes es lineal entre los sitios de medición y no considera ni la variabilidad espacio-temporal de la variable en sí misma (variaciones intraanuales e interanuales) ni la aleatoriedad propia de los fenómenos naturales.

Este método ha sido utilizado comúnmente por su practicidad, pero se lo considera inadecuado en algunos casos, ya que es netamente geométrico, y no tiene en cuenta las características orográficas o de barreras 
naturales que pudieran existir entre las estaciones ni las características convectivas de algunas lluvias, como si lo tendría el método del calculo de la precipitación media areal mediante el trazado de isohietas.

En este trabajo, para determinar los polígonos de Thiessen en el mapa base donde están delimitadas las cuencas, se utilizó el software "QGis" versión 2.18 (http://qgis. osgeo.org) con una subrutina a tal efecto. A partir de conocer la ubicación de las estaciones, trazar los polígonos y recortarlo según los límites de las cuencas, se puede ponderar la aportación espacial de cada sitio en relación al área total de la cuenca.

La precipitación y la evapotranspiración media areal en cada subcuenca se obtuvo mediante la Ecuación 1. Estas variables se calculan para cada mes de la serie de tra- bajo, en forma automática por el programa CHAC. Se utilizan los datos mensuales de todas las estaciones de cada cuenca junto con los pesos de ponderación areal.

Las Figuras 1 y 2 muestran los polígonos de Thiessen resultantes para la precipitación y la evapotranspiración, respectivamente en las 12 cuencas estudiadas de la margen izquierda del río Paraná (las mismas se observan en color marrón). Por su parte en los anexos 1 y 2 se presentan tabulados para cada una de estas cuencas el área correspondiente a cada estación en la que se midió la precipitación o se estimó la evapotranspiración, respectivamente obtenida a partir de los polígonos determinados por este método geométrico, así como el peso de cada una de las estaciones, usado para estimar la $\mathrm{P}$ o la ETP total areal de cada cuenca. 


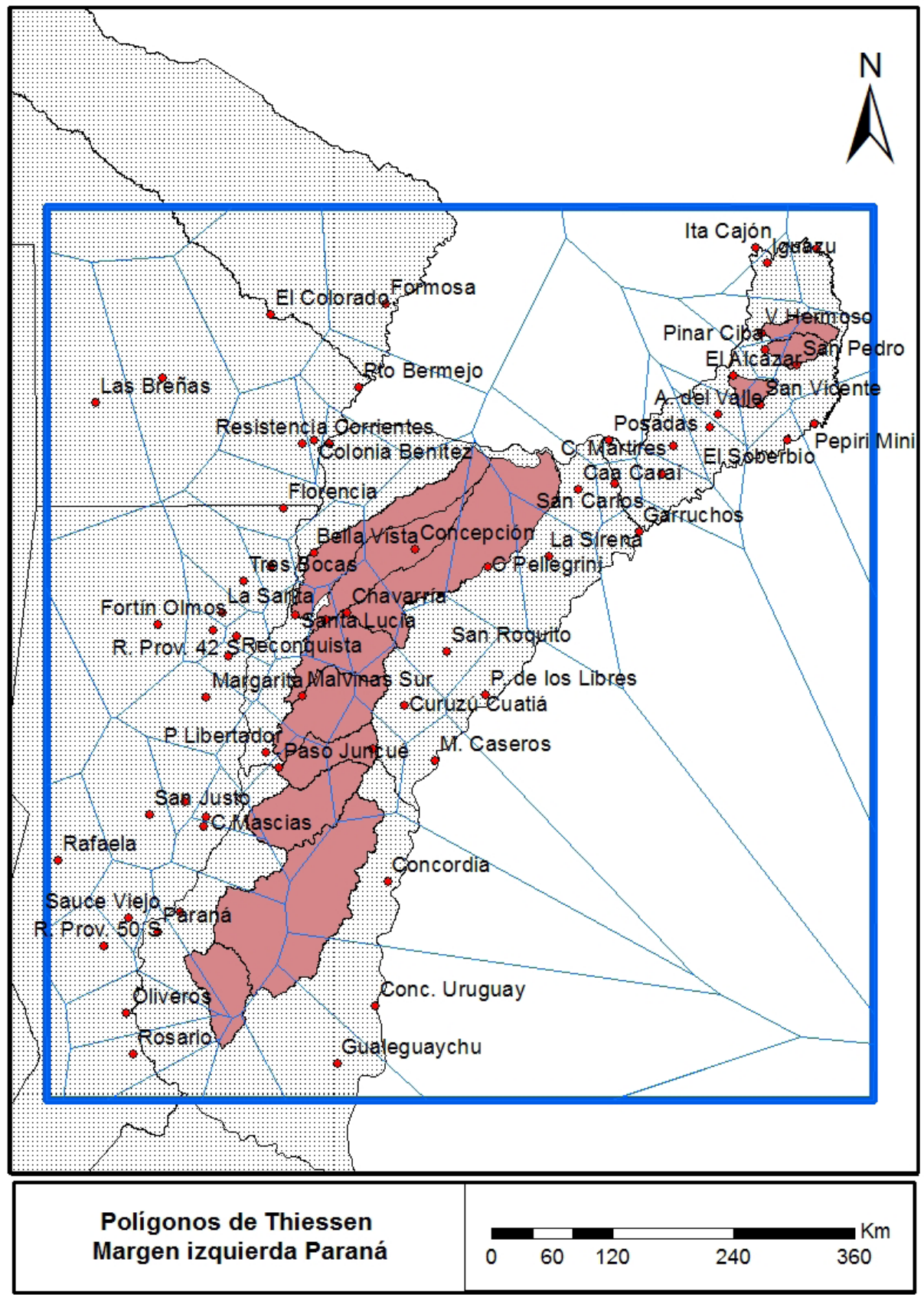

Fig. 1. Trazado de Polígonos de Thiessen para las estaciones de precipitación en las cuencas estudiadas 


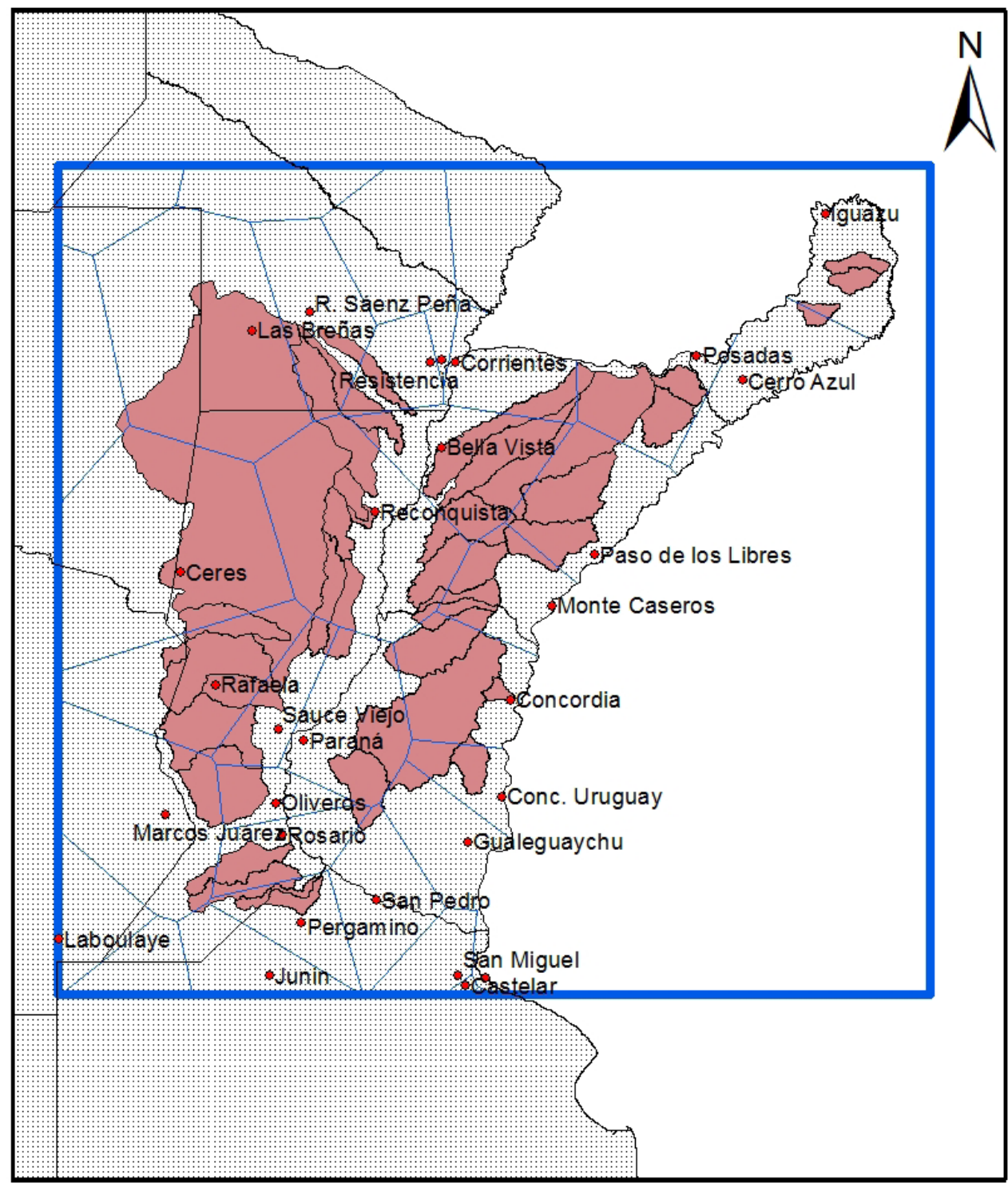

\section{Polígono de Thiessen para ETP}

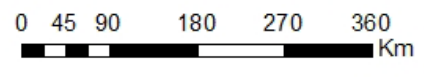

Fig. 2. Trazado de Polígonos de Thiessen para las estaciones de ETP en las cuencas estudiadas. 


\section{Interpolación mediante Kriging}

El kriging es una técnica de interpolación que se basa en el análisis de la estructura geoestadística de variación de la variable estudiada. Es decir se apoya en el conocimiento del comportamiento de la variable en el espacio, con la premisa de que puntos próximos tendrán valores más parecidos que puntos más alejados, ya que asume que una muestra de puntos de una población se encuentra correlacionada en el espacio. El comportamiento de la precipitación, al igual que la evapotranspiración, cumplen con esta premisa, por lo que es posible aplicar esta técnica de obtención de campos espaciales a partir de series de datos puntuales.

La semivariancia es la medida del grado de dependencia espacial entre los puntos de muestreo, su representación gráfica en función de la distancia a un punto está dada por el semivariograma, también llamado variograma, el cual es obtenido a partir de una muestra de la variable (semivariograma experimental), a la que se debe ajustar un modelo teórico.

Para el cálculo del semivariograma experimental se define la media aritmética de todos los cuadrados de las diferencias entre pares de valores experimentales separados una distancia $h$, o lo que es lo mismo, la varianza de los incrementos de la variable regionalizada en las localizaciones separadas una distancia $h$.

$$
\operatorname{Var}\{Z(x+h)-Z(x)\}=2 y(h)
$$

Al estimar la función $\gamma(h)$, es decir la semivariancia en función de la distancia, se obtiene el semivariograma experimental. Para ello se utiliza la expresión:

$$
\gamma(h)=\frac{1}{2 N p(h)} \sum_{i=1}^{N p(h)}\left[Z\left(x_{i}\right)-Z\left(x_{i}+h\right)\right]^{2}
$$

Siendo:

$N p(h)$ : número de pares de puntos separados por la distancia $h$.

$h$ : distancia entre puntos vecinos.

$Z\left(x_{i}\right)$ : valores experimentales.

$x_{i}$ : localizaciones donde son medidos los valores $Z\left(x_{i}\right)$.

Entre los modelos teóricos más comunes que se emplean para ajustar a un semivariograma experimental se encuentran el lineal, el exponencial, el esférico y el gaussiano. Este último modelo señala que el fenómeno evaluado, se más bien continuo, de modo tiene poca variación en los puntos cercanos y suavemente creciente a medida que la distancia aumenta, hasta alcanzar la meseta en la varianza total de la muestra.

De la forma del variograma modelado se extraen los parámetros del modelo que se utilizarán para la interpolación, ellos son la pepita, la meseta y el alcance. La pepita representa el valor de la varianza para la distancia cero, es indicativa de la variabilidad en los datos a pocas distancias (que puede deberse a errores en la obtención de los datos, marcada variabilidad indicativa de procesos fuertemente aleatorios y/o que la separación de los puntos de observación no es suficiente para representar el fenómeno que se está cuantificando); la meseta se define cuando la semivariancia no aumenta más con la distancia y se estabiliza en un valor igual a la varianza de la muestra y; el alcance es la distancia que corresponde a la definición de la meseta.

El análisis de la estructura de variación espacial de la variable, sintéticamente presentado en los párrafos precedentes, es el paso previo ineludible para la aplicación del kriging como método de interpolación. Es decir antes que nada se debe conocer el modelo de variograma ajustado, esto significa: el tipo de modelo y los parámetros del mismo. Además es necesario establecer el tipo de interpolación deseada (puntual o por bloques) y la estrategia adecuada para la se- 
lección de puntos para la interpolación en el rango de búsqueda (que podrá ser circular o elipsoidal dependiendo del comportamiento direccional del variograma). Los datos más allá del alcance, es decir cuando el variograma se vuelve plano, tienen la mínima capacidad predictora y por ende no intervienen en la interpolación. Según ZUCARELLI et al. (2014) "Si el número de valores en el espacio es suficiente, la mejor estrategia para evitar problemas de estacionariedad puede ser disminuir el radio de búsqueda". El resultado final del kriging es un mapa con los valores interpolados de la variable.

Sin embargo, puede resultar que luego del análisis de la estructura de variación espacial el variograma experimental muestre un comportamiento totalmente aleatorio (efecto pepita puro) o lineal. En el primer caso no se deberían trazar mapas con curvas, pues este comportamiento señala que la variable no tiene continuidad espacial (caso común de los fenómenos que se manifiesta a escala muy local, como las fuentes de contaminación puntual en aguas subterráneas o tormentas locales en el caso de precipitaciones diarias). En el segundo, el análisis es- tructural sirve para argumentar la correcta utilización de la interpolación lineal.

La ventaja adicional del kriging sobre los métodos determinísticos, es la estimación de la varianza del error de predicción, lo cual permite además estimar intervalos de confianza para las predicciones realizadas (o eventualmente curvas trazadas). La técnica del kriging es un método de estimación que da el mejor estimador lineal insesgado (BLUE: Best Linear Unbiased Estimator), cuando se cumplen todos los supuestos para su utilización.

En este trabajo para la determinación de la precipitación y evapotranspiración areal de las cuencas, en primer lugar se creó una grilla, sobre la que se sobrepusieron las 12 cuencas estudiadas, lo que se muestra en la Figura 3. A continuación se recortaron las grillas con los contornos de las mismas y por último se calculó la $\mathrm{P}$ o la ETP media con los valores estimados en los puntos dentro de los límites de cada cuenca. Se repite esta operación para los doce meses en todas las cuencas. Se recurrió al software "QGis" versión 2.18 (http://qgis.osgeo.org) para realizar esta tarea. 


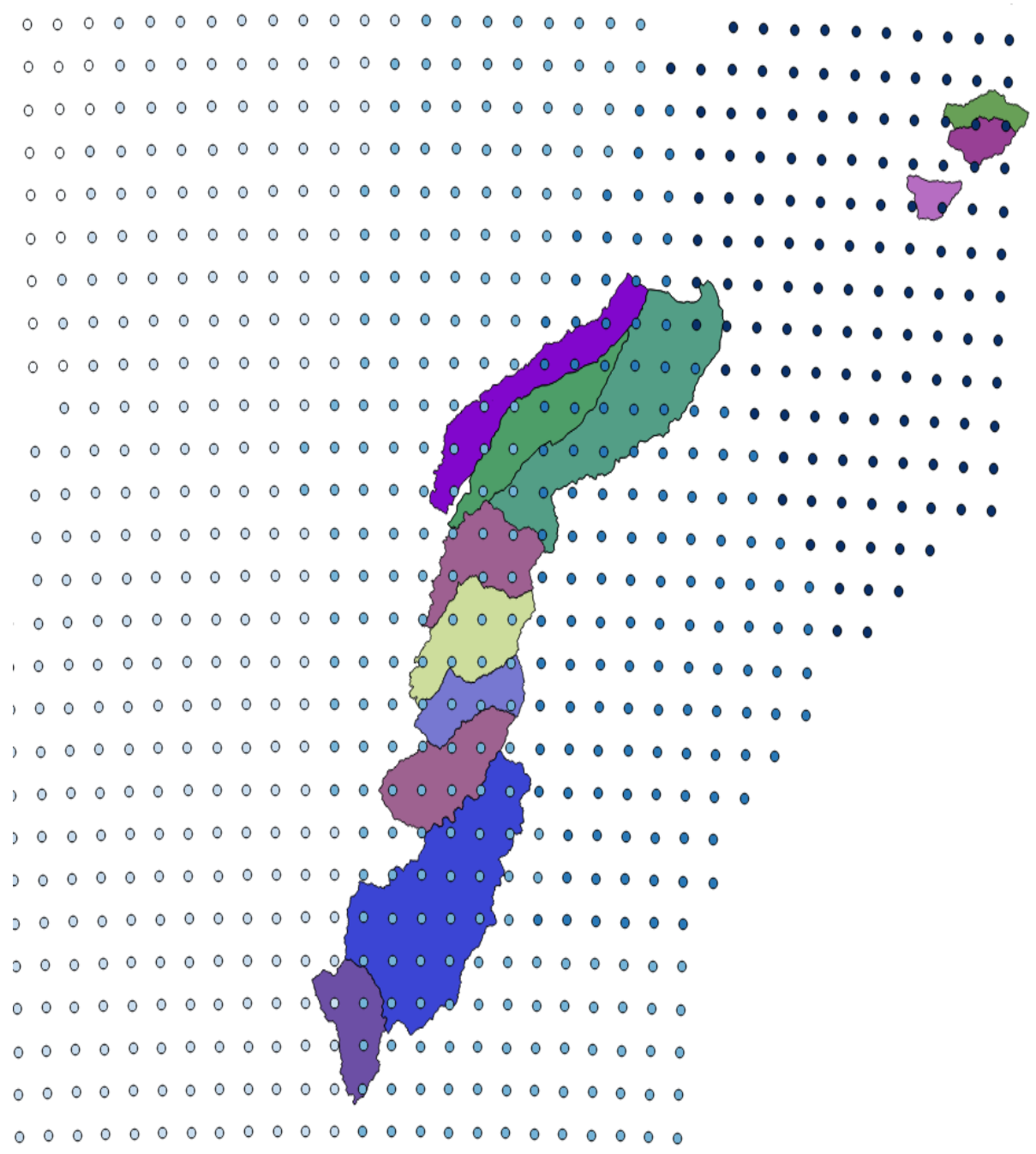

Fig. 3. Ejemplo de grilla y cuencas superpuestas.

\section{RESULTADOS Y DISCUSIÓN}

Las cuencas estudiadas presentan una superficie muy variable que oscila entre 979 km2 en el arroyo Paranay y 15975 en el río Gualeguay. En todas ellas se estimaron campos areales de precipitación y ETP según el método de los polígonos de Thiessen y usando el krigeado ordinario.

\section{Método de polígonos de Thiessen}

Dependiendo de la superficie de la cuenca y las estaciones que registran precipitación dentro o en los alrededores de la misma, el número de polígonos de Thiessen que se utilizaron para la determinación areal de esta variable osciló entre 2 para el arrollo Piray Guazú y 7 para el Río Gualeguay (Fi- 
gura 1 y Anexo 1). Este método es simple de implementar, y ofrece un resultado con base geométrica, ya que se asignan los pesos a las estaciones en función a su ubicación relativa, la distancia entre ellos, y el porcentaje resultante en relación al área total de la cuenca; sin embargo no toma en consideración las características físicas del terreno adyacente, la naturaleza de la variable y si existe otra función de relación espacial que no sea el modelo lineal. En el caso de la margen izquierda del río Paraná, los pesos de los polígonos dentro de cada cuenca pueden presentar magnitudes muy diferentes (Anexo 1).

Para la estimación de la ETP areal se utilizaron los polígonos que se muestran en la Figura 2 y cuyas características se presentan en el Anexo 2. El número de polígonos de Thiessen que se obtuvieron osciló entre solamente 1 para los arroyos Piray Mini y Piray Guazú y 5 para el arroyo Nogayá, lo que se debe a la menor cantidad de estaciones de que se dispone para el cálculo puntual de la ETP en comparación con la precipitación. la contribución de zonas con características distintas dentro de la misma cuenca a la estimación de campos areales está peor definida para la ETP que para la precipitación.

La Tabla 1 muestra los valores medios mensuales y anuales de precipitación y ETP respectivamente, obtenidos por el método de los polígonos de Thiessen en cada cuenca. La precipitación media areal osciló entre 1094 mm para el arroyo Nogoyá y 2028 mm para el Piray Guazú. En Argentina es bien conocido el gradiente norte sur de la precipitación, que se refleja en los resultados obtenidos en este trabajo mediante el método de los polígonos de Thiessen, Por otro lado HÄMMERLY (2017) indica que teniendo en cuanta ambas márgenes del río Paraná, los valores anuales de los campos areales de precipitación oscilan entre 800 y $2000 \mathrm{~mm}$; este resultado concuerda con la presencia de un gradiente este oeste en el monto de las precipitaciones, que se superpone al gradiente norte sur.

La ETP media areal a escala anual se muestra en la Tabla 2, en donde se aprecia que presentó un menor rango de oscilación que la precipitación, oscilando entre 1046 mm en el arroyo Piray Guazú y 1272 $\mathrm{mm}$ en el río Santa Lucia. En las 10 cuencas restantes la ETP oscila solamente entre 1120 y $1250 \mathrm{~mm}$ anuales. Para el conjunto de la cuenca del río Paraná en territorio argentino HÄMMERLY (2017) cita valores anuales de ETP comprendidos entre y 950 y $1300 \mathrm{~mm}$, al tiempo que señala que y contrariamente a lo que ocurre con la precipitación, los campos areales para la ETP no presentan un patrón de gradientes definido, en función de la latitud o de la longitud en las cuencas estudiadas. Sin embargo si se considera toda la cuenca del rio Paraná la ETP tiende a disminuir de este a oeste como consecuencia del aumento de temperatura y de las horas de sol. 


\begin{tabular}{|c|c|c|c|c|c|c|c|c|c|c|c|c|c|c|}
\hline Río o arroyo & Lugar & Sep & Oct & Nov & Dic & Ene & Feb & Mar & Abr & May & Jun & Jul & Ago & Anual \\
\hline Piray Miní & $\begin{array}{l}\text { V. } \\
\text { Hermoso }\end{array}$ & 168 & 236 & 166 & 168 & 162 & 165 & 141 & 175 & 168 & 164 & 111 & 137 & 1960 \\
\hline Piray Guazú & $\begin{array}{l}\text { Pinar } \\
\text { Ciba }\end{array}$ & 172 & 245 & 177 & 174 & 163 & 168 & 141 & 183 & 176 & 172 & 116 & 143 & 2028 \\
\hline Paranay & $\begin{array}{l}\text { El } \\
\text { Alcazar }\end{array}$ & 157 & 232 & 166 & 152 & 158 & 150 & 158 & 163 & 153 & 151 & 124 & 114 & 1876 \\
\hline Santa Lucía & $\begin{array}{l}\text { Santa } \\
\text { Lucia }\end{array}$ & 76 & 149 & 151 & 124 & 129 & 158 & 153 & 178 & 81 & 78 & 54 & 50 & 1383 \\
\hline Corrientes & $\begin{array}{l}\text { Paso } \\
\text { Lucero }\end{array}$ & 91 & 159 & 146 & 124 & 122 & 141 & 140 & 173 & 98 & 92 & 75 & 62 & 1422 \\
\hline Batel & $\begin{array}{l}\text { Paso } \\
\text { Cerrito }\end{array}$ & 78 & 152 & 156 & 120 & 126 & 163 & 146 & 186 & 90 & 86 & 59 & 54 & 1415 \\
\hline Corriente & $\begin{array}{l}\text { Los } \\
\text { Laureles }\end{array}$ & 82 & 148 & 146 & 122 & 123 & 145 & 138 & 172 & 92 & 84 & 65 & 56 & 1371 \\
\hline Barrancas & $\begin{array}{l}\text { P. La } \\
\text { Llana }\end{array}$ & 67 & 123 & 120 & 121 & 127 & 130 & 148 & 158 & 76 & 63 & 42 & 39 & 1214 \\
\hline Guayquiraró & $\begin{array}{l}\text { Paso } \\
\text { Juncué }\end{array}$ & 69 & 126 & 130 & 132 & 137 & 141 & 158 & 162 & 82 & 63 & 39 & 41 & 1280 \\
\hline Feliciano & $\begin{array}{l}\text { Paso } \\
\text { Medina }\end{array}$ & 66 & 123 & 134 & 133 & 142 & 142 & 161 & 163 & 81 & 54 & 35 & 40 & 1272 \\
\hline Nogoyá & $\begin{array}{l}\text { Ruta } \\
\text { Pcial } 11\end{array}$ & 59 & 118 & 117 & 128 & 114 & 130 & 154 & 113 & 59 & 40 & 29 & 34 & 1094 \\
\hline Gualeguay & $\begin{array}{l}\text { Ros. del } \\
\text { Tala }\end{array}$ & 77 & 119 & 129 & 127 & 124 & 132 & 149 & 133 & 82 & 61 & 44 & 45 & 1221 \\
\hline
\end{tabular}

Tabla 1. Precipitación media mensual areal $(\mathrm{mm})$ en cuencas de la margen izquierda del río Paraná, según el método de Thiessen (1970-71 al 2009-10). 


\begin{tabular}{|l|l|l|l|l|l|l|l|l|l|l|l|l|l|l|}
\hline Río o arroyo & Lugar & Sep & Oct & Nov & Dic & Ene & Feb & Mar & Abr & May & Jun & Jul & Ago & Anual \\
\hline Piray Miní & V. Hermoso & 82 & 111 & 135 & 154 & 160 & 125 & 110 & 73 & 51 & 36 & 42 & 61 & 1139 \\
\hline Piray Guazú & Pinar Ciba & 75 & 100 & 116 & 125 & 129 & 112 & 105 & 77 & 54 & 40 & 49 & 65 & 1046 \\
\hline Paranay & El Alcazar & 82 & 108 & 126 & 140 & 142 & 120 & 113 & 81 & 58 & 44 & 52 & 69 & 1135 \\
\hline Santa Lucía & Santa Lucia & 94 & 124 & 141 & 161 & 164 & 135 & 123 & 85 & 64 & 48 & 57 & 75 & 1272 \\
\hline Corrientes & Paso Lucero & 90 & 120 & 138 & 157 & 159 & 131 & 120 & 86 & 63 & 48 & 56 & 74 & 1241 \\
\hline Batel & Paso Cerrito & 93 & 123 & 140 & 160 & 162 & 132 & 122 & 83 & 62 & 47 & 55 & 73 & 1250 \\
\hline Corriente & Los Laureles & 90 & 119 & 137 & 156 & 158 & 130 & 119 & 83 & 62 & 47 & 54 & 72 & 1227 \\
\hline Barrancas & P. La Llana & 86 & 116 & 135 & 157 & 161 & 129 & 115 & 76 & 54 & 40 & 47 & 66 & 1181 \\
\hline Guayquiraró & Paso Juncué & 85 & 114 & 134 & 156 & 161 & 128 & 115 & 75 & 53 & 39 & 46 & 64 & 1169 \\
\hline Feliciano & Paso Medina & 78 & 108 & 131 & 156 & 161 & 125 & 113 & 73 & 49 & 35 & 40 & 58 & 1129 \\
\hline Nogoyá & Ruta Pcial 11 & 85 & 116 & 140 & 164 & 170 & 133 & 119 & 79 & 56 & 40 & 46 & 64 & 1211 \\
\hline Gualeguay & Ros. del Tala & 78 & 107 & 131 & 154 & 160 & 125 & 112 & 73 & 50 & 36 & 41 & 58 & 1123 \\
\hline
\end{tabular}

Tabla 2. Evapotranspiración potencial media mensual areal $(\mathrm{mm})$ en cuencas de la margen izquierda del río Paraná, según el método de Thiessen (1970-71 al 2009-10).

\section{Método de Kriging}

Como se indicó en la sección anterior, en la práctica el método de kriging se inició ajustando modelos de semivariogramas a los semivariogramas muestrales; estos viene definidos por los valores de semivariancia frente a la distancia y fueron obtenidos para todas las series mensuales medias de precipitación y ETP para el período de 1970-2010, que, por tanto, están conformada por los datos de todas las estaciones disponibles. Luego se diseñó una grilla a partir del semivariograma obtenido para cada mes y finalmente se confeccionaron los mapas con las isolíneas, en este caso isohietas e isolíneas de ETP, con una equidistancia preestablecida. Los puntos de la grilla poseen valores en tres coordenadas, que en este caso se corresponden con la latitud, longitud y valor de la variable a interpolar (P o ETP). Los puntos en donde se efectúan las interpolaciones están separados por distancias regulares (Figura 3) y no necesariamente coincidentes con la ubicación de las estaciones. A partir de la grilla también es posible rasterizar el área, ya que cada punto de la grilla representa en el espacio, un valor de la variable.

\section{Estructura espacial y krigeado de la precipi- tación}

Para los datos de precipitación media mensual de cada estación de la serie 19702010 se obtuvieron 12 grillas mensuales, donde los puntos representan en el espacio 
la precipitación media del mes para toda la serie. La Tabla 3 presenta los parámetros de los semivariogramas ajustados gráficamente a las series de precipitación para cada mes. Se comprueba que para todos los meses se ajustó un modelo Gaussiano al semivariograma experimental. A modo de ejemplo, la Figura 4 presenta el variograma ajustado a la muestra para el mes de setiembre, en donde las medidas del eje de ordenadas son en $\mathrm{mm}^{2}$ y la distancia en el eje de abscisas en grados. No obstante durante los meses más fríos, entre mayo y octubre, ambos inclusive fue necesario retirar una tendencia, previamente al cálculo del semivariograma muestral; por el contrario en los meses más cálidos, de noviembre a abril, los modelo de semivariograma de tipo gaussiano pudieron ajustarse directamente a las series de datos de precipitación disponibles sin retirar tendencia.

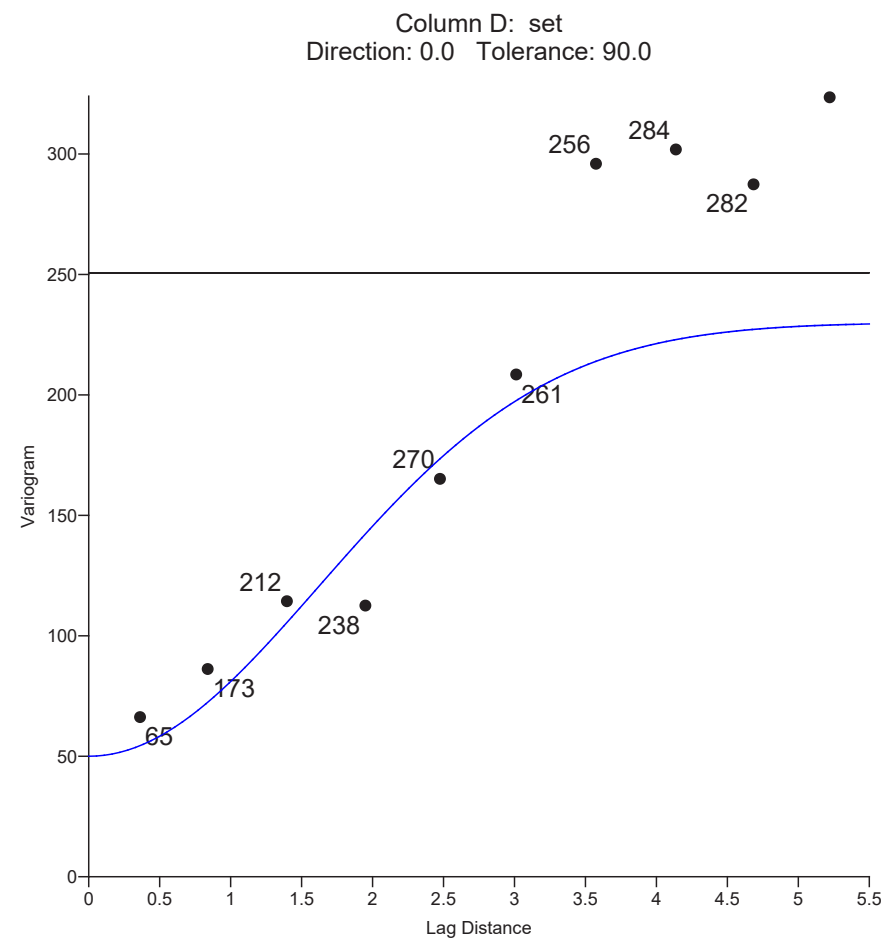

Fig. 4. Ajuste del variograma para la precipitación del mes de setiembre.

Los variogramas del modelo Gaussiano señalan que el fenómeno evaluado, en este caso precipitación mensual, tiene un comportamiento de poca variación, es decir son relativamente continuos cerca del origen, lo que denota analogía en los puntos cercanos y una variación suavemente creciente a medida que la distancia aumenta (CARRERA y SAMPER, 1990). El fenómeno de la llu- via cumple este comportamiento, ya que se caracteriza por una cierta continuidad entre localidades próximas, excepto durante períodos tormentosos, por eso es correcto que éste sea el modelo que ajuste a los datos.

De cuerdo con los datos de la Tabla 3 presentaron un efecto pepita de magnitud variable, que oscila entre 50 y $110 \mathrm{~mm}^{2}$, pero siempre inferior al valor de la varianza 
estructural. En términos relativos el efecto pepita tiende a ser más elevado en los meses en que se retiró tendencia y particularmente entre mayo y agosto. El efecto pepita puede ser consecuencia de una red de muestreo con una densidad demasiado baja para caracterizar la variabilidad espacial a pequeñas distancias o bien de la imprecisión en la medida de los datos disponibles. El al- cance de los semivariogramas gaussianos a los que se ajustó la dependencia espacial de la precipitación varió entre 2,0 y 3,5 grados, es decir entre 223,4 y $491 \mathrm{~km}$; en general el alcance suele ser inferior durante los meses de mayo a setiembre. Los datos medidos en estaciones que están a una distancia inferior al alcance no son independientes entre sí de acuerdo con la teoría geoestadística.

\begin{tabular}{|l|l|l|l|l|l|l|l|l|l|l|l|l|}
\hline Meses & Sep & Oct & Nov & Dic & Ene & Feb & Mar & Abr & May & Jun & Jul & Ago \\
\hline Tendencia & Si & si & no & no & no & no & no & no & si & si & si & si \\
\hline Modelo & Gauss & Gauss & Gauss & Gauss & Gauss & Gauss & Gauss & Gauss & Gauss & Gauss & Gauss & Gauss \\
\hline Pepita & 50 & 110 & 80 & 110 & 80 & 90 & 110 & 100 & 50 & 65 & 50 & 50 \\
\hline Meseta & 180 & 290 & 450 & 290 & 290 & 320 & 270 & 130 & 110 & 75 & 7.5 & 75 \\
\hline $\begin{array}{l}\text { A lcance } \\
\left({ }^{\circ}\right)\end{array}$ & 2.3 & 2.5 & 3.0 & 3.2 & 2.5 & 3.0 & 2.5 & 3.5 & 2.3 & 2.3 & 2.0 & 2.3 \\
\hline
\end{tabular}

Tabla 3. Parámetros de ajuste a los semivariogramas gaussianos para las precipitaciones mensuales (1970-71 al 2009-10).

La precipitación areal se estimó a partir del semivariograma correspondiente, se construyeron las grillas para cada mes. Para ello, en lugar de trazado de mapas, se optó por rasterizar el área y tener un campo de precipitación en el espacio. La Tabla 4 presenta los valores de precipitación mensual media areal para las 12 subcuencas de la margen izquierda del rio Paraná en el período 1970-2010. Los valores anuales de precipitación areal estimados por kriging oscilaron entre 1731 en el arroyo Paranay y $1066 \mathrm{~mm}$ en el arroyo Nogoyá, poniendo de manifiesto una vez más el gradiente norte sur en la distribución de precipitaciones. 


\begin{tabular}{|c|c|c|c|c|c|c|c|c|c|c|c|c|c|c|}
\hline Río o arroyo & Lugar & Sep & Oct & Nov & Dic & Ene & Feb & Mar & Abr & May & Jun & Jul & Ago & Anual \\
\hline Piray Miní & $\begin{array}{l}\text { V. } \\
\text { Hermoso }\end{array}$ & 134.1 & 195.7 & 153.3 & 151.6 & 156.6 & 145.9 & 123.3 & 156.5 & 152.6 & 132.6 & 97.1 & 103.3 & 1703 \\
\hline Piray Guazú & $\begin{array}{l}\text { Pinar } \\
\text { Ciba }\end{array}$ & 137.0 & 198.8 & 153.5 & 152.2 & 156.5 & 146.7 & 125.8 & 158.9 & 152.3 & 134.7 & 100.5 & 105.2 & 1722 \\
\hline Paranay & $\begin{array}{l}\text { El } \\
\text { Alcazar }\end{array}$ & 137.4 & 199.9 & 154.8 & 152.9 & 157.0 & 147.4 & 130.4 & 165.7 & 145.4 & 133.7 & 102.8 & 103.7 & 1731 \\
\hline Santa Lucía & $\begin{array}{l}\text { Santa } \\
\text { Lucia }\end{array}$ & 71.4 & 133.5 & 140.9 & 132.3 & 135.6 & 140.2 & 140.9 & 165.4 & 82.5 & 69.4 & 50.7 & 48.8 & 1312 \\
\hline Corrientes & $\begin{array}{l}\text { Paso } \\
\text { Lucero }\end{array}$ & 86.5 & 146.9 & 139.5 & 131.3 & 131.3 & 139.3 & 135.2 & 169.1 & 95.5 & 82.3 & 66.8 & 61.0 & 1385 \\
\hline Batel & $\begin{array}{l}\text { Paso } \\
\text { Cerrito }\end{array}$ & 72.5 & 132.7 & 140.1 & 130.0 & 132.1 & 140.0 & 139.6 & 166.0 & 83.2 & 69.9 & 51.7 & 48.9 & 1307 \\
\hline Corriente & $\begin{array}{l}\text { Los } \\
\text { Laureles }\end{array}$ & 79.5 & 138.2 & 138.1 & 130.2 & 131.3 & 139.6 & 137.2 & 166.0 & 88.9 & 76.9 & 58.8 & 54.4 & 1339 \\
\hline Barrancas & $\begin{array}{l}\text { P. La } \\
\text { Llana }\end{array}$ & 67.2 & 119.4 & 127.1 & 126.4 & 130.5 & 136.5 & 140.3 & 152.8 & 76.4 & 59.8 & 43.3 & 41.6 & 1221 \\
\hline Guayquiraró & $\begin{array}{l}\text { Paso } \\
\text { Juncué }\end{array}$ & 68.2 & 118.6 & 124.9 & 125.4 & 130.9 & 134.1 & 141.0 & 149.5 & 77.5 & 59.7 & 43.6 & 42.4 & 1215 \\
\hline Feliciano & $\begin{array}{l}\text { Paso } \\
\text { Medina }\end{array}$ & 66.6 & 114.6 & 122.2 & 125.3 & 129.6 & 131.4 & 140.6 & 143.1 & 75.1 & 56.4 & 40.8 & 41.3 & 1187 \\
\hline Nogoyá & $\begin{array}{l}\text { Ruta } \\
\text { Pcial } 11\end{array}$ & 58.4 & 107.9 & 110.8 & 121.5 & 117.4 & 122.6 & 136.3 & 112.2 & 62.0 & 44.2 & 34.8 & 38.2 & 1066 \\
\hline Gualeguay & $\begin{array}{l}\text { Ros. del } \\
\text { Tala }\end{array}$ & 68.5 & 111.5 & 118.4 & 123.7 & 124.4 & 127.2 & 137.1 & 130.6 & 73.1 & 54.3 & 41.4 & 42.2 & 1152 \\
\hline
\end{tabular}

Tabla 4. Precipitación mensual media areal $(\mathrm{mm})$ en cuencas de la margen izquierda del río Paraná, según el método de Kriging (1970-71 al 2009-10).

El método tradicional de ponderación con polígonos de Thiessen, de la precipitación media areal de una cuenca ha proporcionado valores más elevados que el krigeado en 11 de las 12 cuencas estudiadas en la margen izquierda del río Paraná. En dos de las cuencas estudiadas, los arroyos Piray Mini y Piray Guazú, localizadas en la provincia de Misiones las diferencias absolutas entre los dos métodos son superiores a los $250 \mathrm{~mm}$ anuales y en otras dos cuencas, el arroyo Paranay y el río Batel, superan los $100 \mathrm{~mm}$ anuales.

Estos resultados sugieren que, al menos en algunas cuencas, el método empleado para obtener campos areales pueden afectar en una cuantía no desdeñable a la magnitud de los balances hídricos estimados con diferentes modelos.

\section{Estructura espacial y krigeado de la ETP}

La evapotranspiración potencial media areal en cada subcuenca para cada mes se obtuvo por un procedimiento similar. Para los caso de las ETP el ajuste de los semivariogramas experimentales se logró con un modelo lineal para todos los meses. Este modelo sugiere la fuerte influencia de la variación regional de la temperatura en la estimación de la ETP, que se manifiesta con la función lineal representando la tenden- 
cia o deriva regional. La Figura 5 presenta el semivariograma ajustado para el mes de setiembre; las medidas del eje de ordenadas son en $\mathrm{mm}^{2}$ y la distancia en el eje de abscisas en grados

Column D: set

Direction: 0.0 Tolerance: 90.0

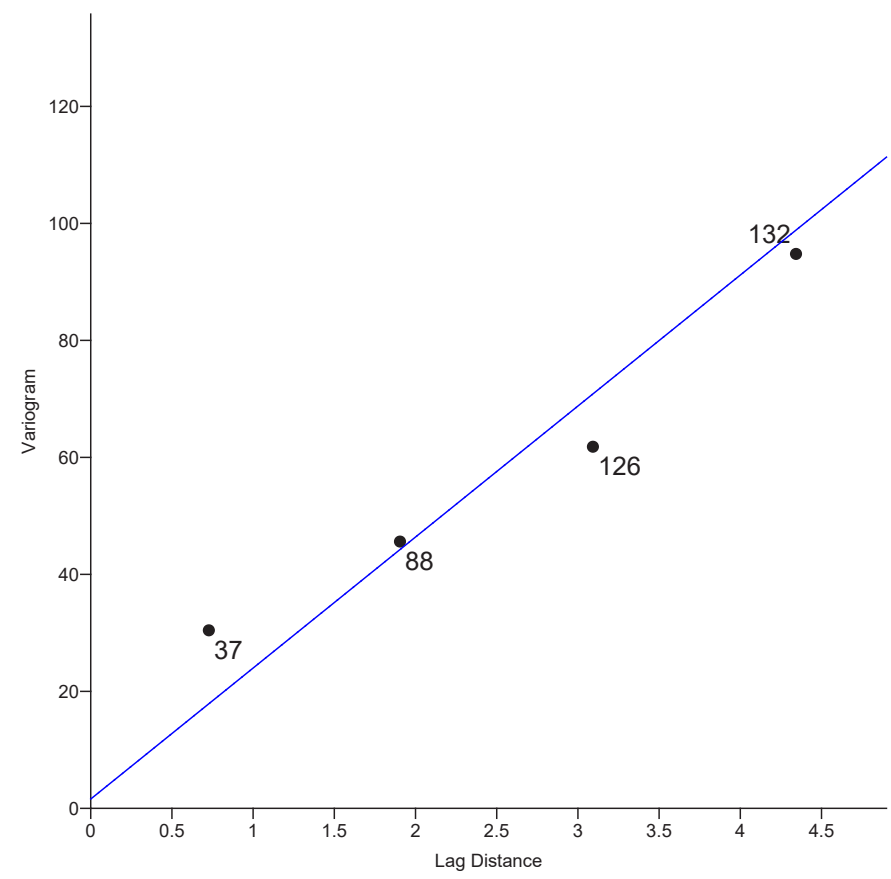

Fig. 5. Ajuste del variograma para la ETP del mes de setiembre.

La Tabla 5 muestra los tipos de semivariogramas y los parámetros de los mismos que fueron adoptados y que se usaron en la confección de las grillas para interpolar los valores mensuales medios areales de ETP. Durante todos los meses del año los semivariogramas muestrales de ETP se ajustaron a un modelo lineal. El mismo presenta valores de efecto pepita que oscilan entre 0 y $25,8 \mathrm{~mm}^{2}$, es decir de una magnitud inferior a los obtenidos para la precipitación. El modelo lineal obtenido para los datos de ETP pone de manifiesto una tendencia al aumento de las horas de sol y por tan- to de la radiación solar según un gradiente este oeste. A pesar de la menor densidad de estaciones en que se ha estimado la ETP, la misma presenta valores de efecto pepita inferiores a los de la precipitación; esto sugiere una menor variabilidad espacial de la ETP en relación con la $\mathrm{P}$ a distancias relativamente pequeñas. La variancia de la precipitación también se presenta en la Tabla 5 , dado que supone un valor de referencia a partir del cual el modelo de dependencia espacial de tipo lineal puede dejar de ser válido al llegar a un valor que corresponde a la meseta del mismo. 


\begin{tabular}{|l|c|c|c|c|c|c|c|c|c|c|c|c|}
\hline \multicolumn{1}{|c|}{ Meses } & Sep & Oct & Nov & Dic & Ene & Feb & Mar & Abr & May & Jun & Jul & Ago \\
\hline Modelo & Lineal & Lineal & Lineal & Lineal & Lineal & Lineal & Lineal & Lineal & Lineal & Lineal & Lineal & Lineal \\
\hline Pepita & 1.6 & 0 & 4.5 & 0 & 13.1 & 10.6 & 25.8 & 7.4 & 8.1 & 8.2 & 7.0 & 9.6 \\
\hline $\begin{array}{l}\text { Varianza } \\
\left(\mathrm{mm}^{2}\right)\end{array}$ & 135.8 & 161.8 & 125.2 & 112.3 & 96.9 & 54.7 & 96.4 & 76.6 & 81.6 & 59.4 & 99.9 & 125.5 \\
\hline
\end{tabular}

Tabla 5. Efecto pepita de los semivariogramas lineales para las ETP mensuales y varianza de las mismas (197071 al 2009-10).

En la Tabla 6 se presentan los valores de evapotranspiración mensual media areal para las 12 subcuencas de la margen izquierda del río Paraná en el período 1970-2010. Las cifras a escala anual oscilaron entre $1108 \mathrm{~mm}$ para el arroyo Piray Miní y 1247 mm para el río Santa Lucía, lo que una vez más pone de manifiesto un rango de oscilación inferior de la ETP comparado con la precipitación.

Los valores de ETP estimados según los polígonos de Thiessen y kriging presentan diferencias absolutas anuales que, en general son de pequeña magnitud, inferiores a los obtenidos para la precipitación. Además, el método de los polígonos de Thiessen no siempre proporciona valores más elevados que el de kriging; de hecho los polígonos de Thiessen solo estiman valores más elevados de EPP en 4 de las 12 estaciones. Las mayores diferencias corresponden al arroyo Paray Guazú e donde la ETP estimada según los polígonos de Thiessen a escala anual es 67 veces inferior a la obtenida por krigeado; por el contrario en el arroyo Nogoyá el método de los polígonos de Thiessen supera en $56 \mathrm{~mm}$ anuales a los resultados obtenidos por kriging. En el resto de las estaciones las diferencias anuales son inferiores a $40 \mathrm{~mm}$.

Cuando se comparan las diferencias de P y ETP obtenidas por los dos métodos empleados en este trabajo a escala mensual las diferencias relativas pueden ser todavía más elevadas que las obtenidas a escala anual. Se comprueba entonces que surge la necesidad de estudiar, validar y difundir metodologías, que permitan estimar campos de precipitaciones y evapotranspiración más confiables para lograr mejores resultados en los balances (HÄMMERLY et al., 2017). 


\begin{tabular}{|c|c|c|c|c|c|c|c|c|c|c|c|c|c|c|}
\hline Río o arroyo & Lugar & Sep & Oct & Nov & Dic & Ene & Feb & Mar & Abr & May & Jun & Jul & Ago & Anual \\
\hline Piray Miní & $\begin{array}{l}\text { V. } \\
\text { Hermoso }\end{array}$ & 78.7 & 104.3 & 121.2 & 132.8 & 137.6 & 118.6 & 112.5 & 80.7 & 57.7 & 44.0 & 51.7 & 68.0 & 1108 \\
\hline Piray Guazú & $\begin{array}{l}\text { Pinar } \\
\text { Ciba }\end{array}$ & 79.6 & 106.5 & 122.2 & 132.9 & 137.7 & 118.6 & 113.1 & 80.8 & 58.1 & 44.0 & 52.0 & 68.0 & 1113 \\
\hline Paranay & $\begin{array}{l}\text { El } \\
\text { Alcazar }\end{array}$ & 81.6 & 108.1 & 125.8 & 140.4 & 143.2 & 121.6 & 114.3 & 82.2 & 59.0 & 44.8 & 52.7 & 69.3 & 1143 \\
\hline Santa Lucía & $\begin{array}{l}\text { Santa } \\
\text { Lucia }\end{array}$ & 92.3 & 122.3 & 139.5 & 160.7 & 161.8 & 132.1 & 119.8 & 83.6 & 61.8 & 46.0 & 54.5 & 72.9 & 1247 \\
\hline Corrientes & $\begin{array}{l}\text { Paso } \\
\text { Lucero }\end{array}$ & 91.1 & 121.0 & 139.1 & 160.1 & 160.9 & 131.3 & 119.1 & 84.3 & 61.7 & 46.0 & 54.6 & 72.8 & 1242 \\
\hline Batel & $\begin{array}{l}\text { Paso } \\
\text { Cerrito }\end{array}$ & 91.6 & 121.7 & 139.4 & 161.0 & 162.2 & 131.9 & 119.5 & 83.1 & 61.2 & 45.5 & 53.8 & 72.2 & 1243 \\
\hline Corriente & $\begin{array}{l}\text { Los } \\
\text { Laureles }\end{array}$ & 90.6 & 120.5 & 138.7 & 160.0 & 161.4 & 131.3 & 119.0 & 83.1 & 60.8 & 45.3 & 53.5 & 71.8 & 1236 \\
\hline Barrancas & $\begin{array}{l}\text { P. La } \\
\text { Llana }\end{array}$ & 85.7 & 115.6 & 135.8 & 157.7 & 162.3 & 129.6 & 116.7 & 77.9 & 54.2 & 41.2 & 47.8 & 66.3 & 1191 \\
\hline Guayquiraró & $\begin{array}{l}\text { Paso } \\
\text { Juncué }\end{array}$ & 84.2 & 114.0 & 135.0 & 157.1 & 162.5 & 128.8 & 115.8 & 76.6 & 55.8 & 39.8 & 46.0 & 64.5 & 1180 \\
\hline Feliciano & $\begin{array}{l}\text { Paso } \\
\text { Medina }\end{array}$ & 82.6 & 112.6 & 134.6 & 157.0 & 162.9 & 128.4 & 115.0 & 75.5 & 53.0 & 38.7 & 44.5 & 62.9 & 1168 \\
\hline Nogoyá & $\begin{array}{l}\text { Ruta } \\
\text { Pcial } 11\end{array}$ & 81.4 & 111.4 & 136.7 & 158.7 & 164.1 & 127.5 & 111.9 & 73.6 & 51.0 & 36.6 & 42.0 & 60.3 & 1155 \\
\hline Gualeguay & $\begin{array}{l}\text { Ros. } \\
\text { del Tala }\end{array}$ & 80.1 & 109.9 & 134.0 & 156.7 & 163.2 & 127.1 & 113.0 & 73.8 & 51.0 & 36.8 & 42.1 & 60.1 & 1148 \\
\hline
\end{tabular}

Tabla 6. Evapotranspiración potencial media areal $(\mathrm{mm})$ en cuencas de la margen izquierda del río Paraná, según el método de Kriging (1970-71 al 2009-10).

\section{CONCLUSIONES}

Al emplear el método de los polígonos de Thiessen que se utilizaron para la evaluación de campos areales de precipitación y ETP en 12 cuencas de la margen izquierda del río Paraná con la información meteorológica disponible se puso de manifiesto que se dispone de menos estaciones en las que se puede calcular la ETP en comparación con aquellas en que se registra la precipitación. Por lo tanto, la contribución de zonas con características distintas dentro de la misma cuenca a la estimación de campos areales está peor definida para la ETP que para la precipitación.
Los datos medidos mensuales de precipitación y ETP presentaron dependencia espacial que fue descrita por semivariogramas de tipo lineal y gaussiano, respectivamente. Los semivariogramas de tipo gaussiano que describen la dependencia espacial de la precipitación presentaron un efecto pepita de magnitud variable pero siempre inferior al valor de la varianza estructural; en términos relativos dicho efecto pepita tiende a ser más elevado en los meses de invierno y particularmente entre mayo y agosto. El alcance de los semivariogramas gaussianos osciló entre 223,4 y $491 \mathrm{~km}$. Los semivariograms de tipo lineal obtenidos para la ETP presentaron efectos 
pepita inferiores a los obtenidos para la precipitación

El método tradicional de ponderación con polígonos de Thiessen, de la precipitación media areal de una cuenca ha proporcionado valores más elevados que el krigeado en 11 de las 12 cuencas estudiadas en la margen izquierda del río Paraná. En dos de las cuencas estudiadas, localizadas en la provincia de Misiones las diferencias absolutas de preciptación estimadas por estos dos métodos son superiores a los $250 \mathrm{~mm}$ anuales y en otras dos cuencas superan los $100 \mathrm{~mm}$ anuales. Los valores de ETP estimados según los polígonos de Thiessen y krigeado presentan diferencias absolutas que en general son de pequeña magnitud, de modo que solo en dos cuencas alcanzan valores comprendidos entre 50 y $70 \mathrm{~mm}$ anuales, aproximadamente; además el método de los polígonos de Thiessen proporciona valores más elevados en 4 de las 12 estaciones, pero no en las restantes. Estos resultados, en particular los referidos a la precipitación, sugieren que el método empleado para obtener campos areales pueden afectar en una cuantía no desdeñable a la magnitud de los balances hídricos estimados con diferentes modelos. Surge entonces la necesidad de estudiar, validar y difundir metodologías, que permitan estimar campos de precipitaciones y evapotranspiración más confiables y lograr mejores resultados en los balances.

\section{REFERENCIAS}

BARBOSA LANDIM, P. 1997. Análise Estadística de dados geológicos. UNESP Editora. San Pablo, Brasil. 226 pp.

CARRERA, J., SAMPER, J. 1985. Apuntes del Curso sobre métodos geoestadísticos aplicados a la Hidrología Subterránea. Escuela Técnica Superior de Ingenieros de caminos, canales y puertos de Valencia.
CARRERA, J., SAMPER, J. 1990. Geoestadística: Aplicaciones a la $\mathrm{Hi}$ drología Subterránea. CIMNE. Universidad de Barcelona. 484 pp.

CEDEX. 2012. Manual de Usuario CHAC. Madrid, España. 76 pp.

CHOW, V.T., MAIDMENT, D., MAYS, L. 1996 Hidrología aplicada. McGraw-Hill, Santa Fe de Bogotá, 584 pp.

CORTÉZ, A., OVALLES, F.A., RODRÍGUEZ, M.F., REY, J.C., NÚÑEZ, M.C. 2005. Análisis geoestadístico de la variabilidad temporal, a una escala anual y mensual, de parámetros climáticos en un campo experimental, estado Aragua, Venezuela. Agronomía Tropical, 55 (3), 327-342.

DAMILANO, G., PARIS, M. 2014. Apuntes del Curso Geoestadística. Maestría en Ciencias Agropecuarias. Facultad de Agronomía y Veterinaria, Universidad Nacional de Río Cuarto. Cordoba Argentina. 18 pp.

DEMEY, R.J., PRADERE, R. 1996. Generación de isolíneas de precipitación al sur del Estado de Aragua-Venezuela usando kriging con tendencia externa. Agronomía Tropical, 46(3), 313-333.

GUERRA, F., GÓMEZ, H., GONZÁLEZ, J., ZAMBRANO, Z. 2006. Uso actual de métodos y técnicas para el estudio de la precipitación incluyendo plataformas SIG. Geoenseñanza, 11(1), 97-1

HÄMMERLY, R., PARIS, M., PAOLI, C. 2012. Análisis de la estructura espacial de una tormenta con métodos geoestadísticos. XXV Congreso Latinoamericano de Hidráulica, San José, Costa Rica.

HÄMMERLY, R. 2017. Variabilidad regional de los componentes del balance hídrico. Tesis doctoral. Universidade da Coruña (UDC), 176 pp. 
HÄMMERLY, R., PAOLI, C., DUARTE O.C. 2018. Distribución de la precipitación y la evapotranspiración en territorio argentino de Cuenca del Plata. Cadernos do Laboratorio Xeolóxico de Laxe, 40, 69 - 102.

ÍNIGUEZ COVARRUBIAS, M., OJEDA BUSTAMANTE, W., DÍAZ DELGADO, C., MAMADOU BÂ, K., MERCADO ESCALANTE, R. 2011. Análisis metodológico de la distribución espacial de la precipitación y la estimación media diaria. Revista mexicana de ciencias agrícolas, 2 (1), 57-69.

IZQUIERDO, T., MÁRQUEZ, A. 2006. Comparación de métodos de interpolación para la realización de mapas de precipitación para el acuífero de IcodCañadas (Tenerife, Islas Canarias). Geogaceta, (40), 307-310.

MATHERON, G. 1971. The theory of regionalized variables and its applications. Les Cahiers du Centre de Morphologie Mathématique, № 5, Ecole des Mines de Paris, France. 211 pp.

MEJÍA, J.F., MESA, O., POVEDA, G., VÉLEZ, J.I., HOYOS, C.D., MANTILLA, R., BOTERO, B. 1999. Distribución espacial y ciclos anual y semianual de la precipitación en Colombia. DYNA Revista de la Facultad de Minas, Universidad Nacional de Colombia, Sede Medellín, (127),7-24.

MEZHER, R.N., MERCURI, P.A. 2009. Análisis geoestadístico de la distribución de eventos de granizo en Argentina. Re- unión Científica de la Asociación Argentina de Geofísicos y Geodestas. 24. Taller de Trabajo de Estaciones Continuas GNSS de América y del Caribe. 1. 2009 04 14-17, Mendoza, AR.

PINEDA CONTRERAS, N., ARELLANO GODOY, R., BECERRA SÁNCHEZ, L., AULAR VILLEGAS, M. E., AZUAJE VALERA, M., PIMENTEL MENDOZA, J., JAIMES CÁRDENAS, E. 2011. Caracterización climática de la microcuenca del Río Monaicito, subcuenca del Río Motatán-Carache. Revista mexicana de ciencias agrícolas, 2(5), 765-771.

QUANTUM GIS DEVELOPMENT TEAM. 2017. Quantum GIS Geographic Information System. Open Source Geospatial Foundation Version 2.18. Project. http://qgis.osgeo.org.

TUCCI, C.E.M. (Ed.) 2000. Hidrología. Ciencia y aplicación. Universidade/ UFRGS - ABRH, 2. ed Porto Alegre, Brasil, 943 pp.

TUCCI, C.E.M. 2002. Regionalização de Vazoes. Editorial de Universidades. Porto Alegre, Brasil, $256 \mathrm{pp}$.

ZUCARELLI, A., PARIS, M., MACOR, J. 2014. Utilización de kriging para la elaboración de curvas isohietas de precipitación mensual en la Provincia de Santa Fe, Argentina. $2^{\text {do }}$ Encuentro de Investigadores en Formación en Recursos Hídricos. Instituto Nacional del Agua (INA). Ezeiza, Buenos Aires, Argentina. 